\title{
A catalog of planetary nebula candidates in the Sculptor spiral galaxy NGC $300^{\star}, \star \star$
}

\author{
M. Peña ${ }^{1}$, J. Reyes-Pérez ${ }^{1}$, L. Hernández-Martínez ${ }^{2}$, and M. Pérez-Guillén ${ }^{1}$ \\ ${ }^{1}$ Instituto de Astronomía, Universidad Nacional Autónoma de México, Apdo. Postal 70264, Méx. D. F., 04510 México, México \\ e-mail: [miriam;jperez;lilihe; jguillen] @astro.unam.mx \\ ${ }^{2}$ Instituto Nacional de Astrofísica, Optica y Electrónica, Tonantzinta, Pue., México
}

Received 26 April 2012 / Accepted 27 August 2012

\begin{abstract}
Aims. [O III] $5007 \AA$ on-band and off-band images, obtained with the Very Large Telescope (VLT) and FORS 2 spectrograph in two zones (center and outskirts) of the spiral galaxy NGC 300, are analyzed searching for emission line objects. In particular we search for planetary nebula $(\mathrm{PN})$ candidates to analyze their distribution and luminosity properties, to perform follow-up spectroscopy, and to study the planetary nebula luminosity function, PNLF.

Methods. In the continuum-subtracted images, a large number of emission line objects were detected. From this sample we selected those objects with stellar appearance and no detectable central star as PN candidates. [O III] $5007 \AA$ instrumental magnitudes were measured and calibrated by using spectrophotometric data from the follow-up spectroscopy.

Results. We have identified more than a hundred PN candidates and many compact HII regions. The PN sample is the largest one reported for this galaxy so far. For all the objects we present coordinates, instrumental [O III] 5007 magnitudes, and apparent nebular [O III 5007 fluxes and magnitudes. The [O III] 5007 observed luminosity function for PNe (PNLF) was calculated for the whole sample and for the central and outskirts samples. The three PNLF are similar within uncertainties. We fit the empirical PNLF to the observed PNLF for all the samples. From our best fit for the whole sample, we derived a maximum value for the apparent magnitudes of $m_{5007}^{\star}=22.019 \pm 0.022$ and obtained a tentative estimate of the distance modulus $m_{5007}-M_{5007}=26.29_{-0.22}^{+0.12}$ mag, which agrees well with the recent value derived from Cepheid stars.
\end{abstract}

Key words. galaxies: individual: NGC 300 - planetary nebulae: general - HII regions - galaxies: distances and redshifts

\section{Introduction}

Planetary nebulae (PNe) constitute the evolutionary end point of low-intermediate mass stars with initial masses in the range of 1-8 $M_{\odot}$. Their central stars are post-AGB objects whose UV radiation ionizes the nebula ejected during the AGB stage. Because PNe emit selectively in a few strong and narrow emission lines, they can be discovered at significant distances within the nearby Universe (at least up to $30 \mathrm{Mpc}$ ). The study of $\mathrm{PNe}$ provides accurate information on the luminosity, age, metallicity, and dynamics of the parent stellar population, and this makes them very useful for testing many theories about the evolution of low-intermediate mass stars and their influence in galaxies.

PNe in external galaxies are also useful as distance indicators through the [O III] $5007 \AA$ planetary nebulae luminosity function (PNLF). Ciardullo et al. (1987) and Jacoby (1989) reported that the bright end of the PNLF seems invariant for all the galaxies they investigated, thus they proposed that the PNLF can be used as distance indicator if a complete PN sample in the two or three brightest magnitudes is obtained. The advantage of this method is that we can see bright PNe in galaxies of all kind of Hubble types, and PNe are easily identified. Although more recently

* Based on observations collected at the European Southern Observatory, VLT, Paranal, Chile, program ID 077.B-0430.

$\star \star$ Table 2 is also available in electronic form at the CDS via anonymous ftp to cdsarc.u-strasbg. fr $(130.79 .128 .5)$ or via http://cdsarc.u-strasbg.fr/viz-bin/qcat?]/A+A/547/A78 there has been some evidence that the standard empirical PNLF does not fit well in some irregular galaxies, such as the cases of the SMC presented by Jacoby \& De Marco (2002), the LMC discussed by Reid \& Parker (2010) and NGC 6822 analyzed by Hernández-Martínez \& Peña (2009), the bright end of the PNLF continues to be a robust secondary distance indicator in all kinds of galaxies (for spiral galaxies see the work by Herrmann et al. 2008).

The Scd spiral galaxy NGC 300 is located in the Sculptor Group at a distance of $1.88 \mathrm{Mpc}$ (distance modulus of $26.37 \pm$ 0.08) as derived from Cepheid stars by Gieren et al. (2005). Owing to its proximity to the Milky Way, this galaxy has been the subject of numerous studies related to its stellar content in the central and outskirt regions. The abundance patterns of its massive stars and HII regions, as well as the radial abundance gradients, have been analyzed recently (Urbaneja et al. 2005; Bresolin et al. 2009 and references therein). Surveys for PNe have been reported by Soffner et al. (1996) and Rizzi et al. (2006), but only Soffner et al. have published a list with the coordinates and characteristics of 34 PN candidates.

The main aims of this work are to present the PN candidates detected with the [O III] $5007 \AA$ on-band, off-band technique, during a deep survey looking for PN candidates in two zones (central and outskirts) of NGC 300 and to investigate the PN population in this galaxy. We also built and analyzed the PNLF. Images were obtained as a "pre-imaging" program for follow-up spectroscopy of a sample of emission line objects. Here we present the results of the imaging and part of the 


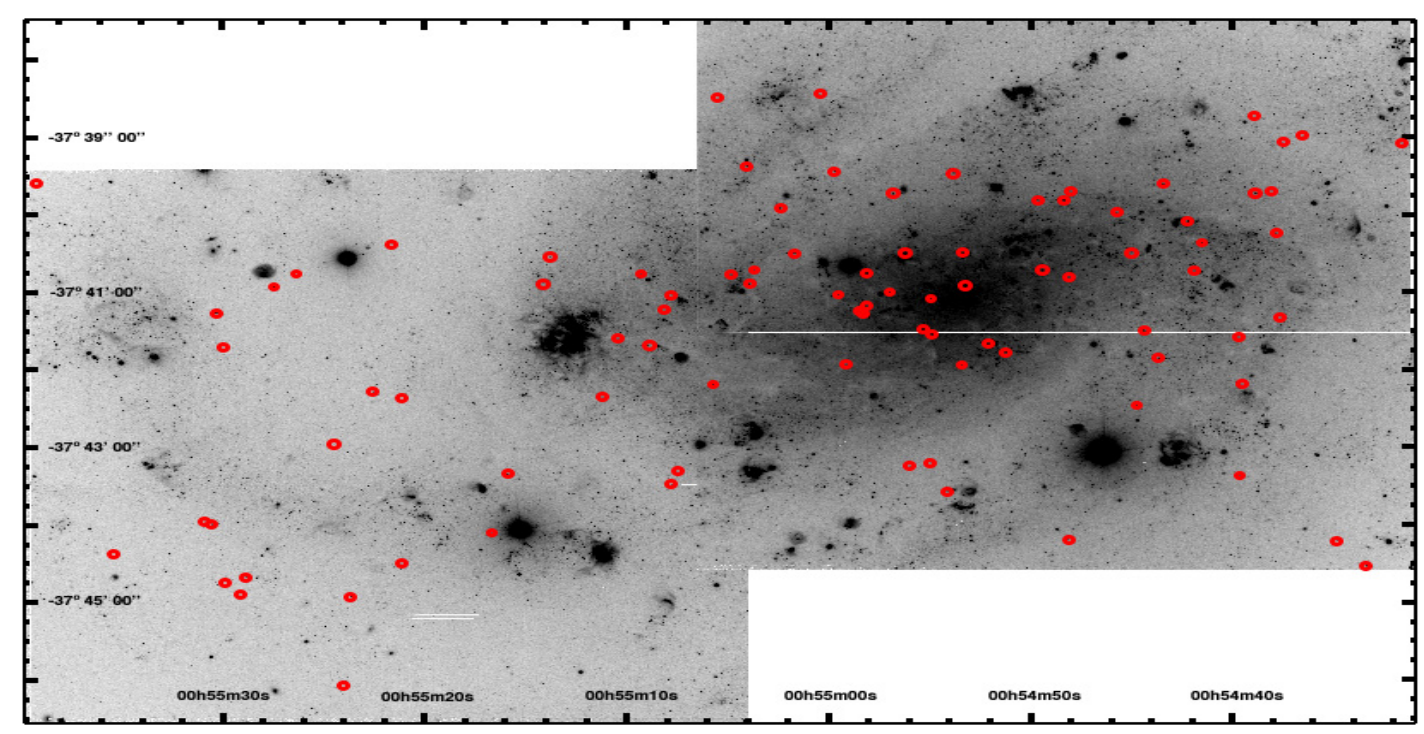

Fig. 1. Images in $\left[\mathrm{O}_{\mathrm{III}}\right] \lambda 5007$ observed for the center and outskirt zones, put together. R.A. and Dec coordinates are marked for J2000. North is up. The detected PN candidates are marked.

spectral data that is used to validate the identification of objects as PNe or HII regions and to calibrate our instrumental [O III] 5007 mag. Complete analysis of the spectroscopy will be presented in a second paper where line fluxes, physical conditions, and chemical compositions of observed objects will be analyzed (Stasińska et al. 2012).

The paper is organized as follows: in Sect. 2 we present the observations and data reduction. In Sect. 3, the criteria to separate $\mathrm{PN}$ candidates from other emitting objects and the obtained samples are described. Also comparative photometry and flux calibration for the detected objects are carried out in this section. In Sect. 4 we discuss the observed PNLF constructed from the $\left[\mathrm{O}_{\mathrm{III}}\right] 5007$ apparent magnitudes and the fit of the empirical PNLF to the observed ones. Our results are summarized in Sect. 5.

\section{Observations and data reduction}

\subsection{Imaging}

[O III] $5007 \AA$ on-band and off-band imaging was obtained in service mode, at the Very Large Telescope, VLT, UT1 (Antu) of the European Southern Observatory (ESO), Paranal, Chile, equipped with the spectrograph FORS 2 (Appenzeller et al. 1998), on 2006-07-04. This constituted the pre-imaging of program ID 077.B-0430. The filters FILT_500_5+85 (on-band filter, central wavelength $5000 \AA$, FWHM $50 \AA$ ) and OIII/6000+52 (off-band filter, central wavelength $5105 \AA$, FWHM $61 \AA$ ) were used.

Two zones were observed. The first one, centered at RA = $00^{\mathrm{h}} 54^{\mathrm{m}} 49.00^{\mathrm{s}}, \mathrm{Dec}=-37^{\circ} 41^{\prime} 02.0^{\prime \prime}$, corresponds to the center of the galaxy, and the second zone is in the outskirts, with central position RA $=00^{\mathrm{h}} 55^{\mathrm{m}} 22.00^{\mathrm{s}}$, Dec $=-37^{\circ} 43^{\prime} 00.0^{\prime \prime}$. For each zone, two on-band images of $375 \mathrm{~s}$ exposure time each, and two off-band exposures of $260 \mathrm{~s}$ each, were acquired. The covered area in each zone is $6.8 \times 6.8 \mathrm{arcmin}^{2}$ (corresponding to the field of view of FORS 2), with a spatial scale of $0.25^{\prime \prime} /$ pix (standard resolution). In Table 1 we list the individual images obtained that, for each position, were slightly dithered for better coverture of the gap between both CCDs of FORS 2.

Images were reduced and calibrated with the normal procedures of the ESO pipeline. That is, reduced images are
Table 1. On-band and off-band images obtained for NGC 300 with FORS 2.

\begin{tabular}{lcc}
\hline \hline \multicolumn{3}{l}{ Central zone, center RA $=00^{\mathrm{h}} 54^{\mathrm{m}} 49.0^{\mathrm{s}}$, Dec $=-37^{\circ} 41^{\prime} 02.0^{\prime \prime}$} \\
\hline frame & filter & $\mathrm{ET}^{1}$ \\
\hline r.FORS2.2006-07-04T07 $4050.519 \& 520$ & FILT_500_5 & 375 \\
r.FORS2.2006-07-04T07 $4745.890 \& 891$ & FILT_500_5 & 375 \\
r.FORS2.2006-07-04T07 54 51.223\&224 & OIII/6000 & 260 \\
r.FORS2.2006-07-04T07 59 52.257\&258 & OIII/6000 & 260 \\
\hline \multicolumn{4}{l}{ Outskirt zone, center RA = 00 ${ }^{\mathrm{h}} 55^{\mathrm{m}} 22.0^{\mathrm{s}}$, Dec $=-37^{\circ} 43^{\prime} 00.0^{\prime \prime}$} \\
\hline r.FORS2.2006-07-04T08 09 36.304 \&305 & FILT_500_5 & 375 \\
r.FORS2.2006-07-04T08 $1711.560 \& 561$ & FILT_500_5 & 375 \\
r.FORS2.2006-07-04T08 24 55.767 \& 768 & OIII/6000 & 260 \\
r.FORS2.2006-07-04T08 29 58.301 \& 302 & OIII/6000 & 260 \\
\hline
\end{tabular}

Notes. ${ }^{(1)}$ Exposure time in seconds.

bias-subtracted, flat-fielded, and astrometrically calibrated, but they are not photometrically calibrated. According to FORS1+2 Users Manual (VLT-MAN-ESO-13100-1543, Issue 3), the astrometric precision is one pixel, equivalent to 0.25 arcsec. During the observations, the seeing conditions were better than 0.9 arcsec and the sky transparency was clear.

Each set of reduced images of Table 1 was first aligned and then recombined using IRAF $^{1}$ routines. The resulting images are equivalent to a $12.5 \mathrm{~min}$ exposure for the [O III] on-band filter and a 8.7 min exposure for the off-band filter. In Fig. 1, we present a combined image showing the central and outskirt [O III] on-band images. There is a small overlap between both zones. The analysis of the images is presented in Sect. 3.

\subsection{Spectroscopy and calibration of imaging}

Follow-up spectroscopy for more than 40 objects (classified as PN candidates and compact HII regions in our imaging) was carried out with the same telescope and instrument (VLT and FORS2 in MXU mode, program ID 077.B-0430(B)) on 1 IRAF is distributed by the National Optical Astronomy
Observatories, which is operated by the Association of Universities for
Research in Astronomy, Inc., under contract to the National Science Foundation. 
2006 August 19-22. Although a complete analysis of the spectrophotometric data will be presented elsewhere, here we describe the observations and present partial results because the spectroscopic data helps to confirm the nature of the analyzed objects (PN or compact HII region), and we use the calibrated [OIII] 5007 fluxes obtained spectroscopically, to calibrate our imaging data (see Hernández-Martínez \& Peña 2009 for a complete description of this procedure).

The grisms 600B, 600RI, and 300I were used to cover a spectral range from about 3600 to $9500 \AA$. Several frames were obtained for each grism. Total exposure times were of about 3.3 to $3.5 \mathrm{~h}$ for spectra with grisms $600 \mathrm{~B}$ and 600RI, and about $1.5 \mathrm{~h}$ for spectra with grism 300I. The slit width was $1^{\prime \prime}$ for all the objects and the spectral resolution varied from about $0.7 \AA$ to $1.2 \AA$. Data were reduced and calibrated using the ESO pipeline and IRAF routines. The standard stars EG274, LDS749B and BMP16274 were observed each night, through a slit of $5^{\prime \prime}$ width, for flux calibration. During the spectroscopic run the sky was clear, and the seeing conditions varied from $0.7^{\prime \prime}$ to $0.9^{\prime \prime}$, however some flux could have been lost in the $1^{\prime \prime}$ slit. The losses can amount to 10-15\%, which should be considered in the uncertainties of our flux calibration.

\section{Analysis and calibration of the images}

To search for emission line objects, the on-band and off-band combined images were scaled (by scaling some stars present in both images), and then they were subtracted. This allows the effect of crowding to be reduced and the emission line objects are more easily detected in the subtracted image. In addition we used the "blinking" technique of the on-band and off-band images, which is very powerful for detecting faint emitting objects by eye. The continuum-subtracted [O III] 5007 images show numerous extended and compact emitting objects, from which we selected all the possible PN candidates and some compact HII regions. The criteria for distinguishing between both type of objects are described in the next section.

\subsection{Separating PN candidates from other emission line objects}

The PN candidates were selected following the prescriptions given in Peña et al. (2007) and in references there; that is, PN candidates should be point-like objects (at the distance of NGC 300, a 1 pc diameter nebula would appear as a $0.1^{\prime \prime}$ size object, thus, not resolved) and the central star should not be detected. This is not the case for central stars of HII regions, because for a distance modulus of $26.37 \mathrm{mag}$, an O9.5 V-B0 V star (the faintest able of producing a very low excitation HII region), possessing an absolute magnitude $M_{\mathrm{V}} \sim-3.90$ (Martins et al. 2005), has an apparent magnitude $V \sim 22.5 \mathrm{mag}$, which translates into $m(5007) \sim 25.6 \mathrm{mag}$ for the narrow-band filter we used. Actually the observed magnitude would be about 26 mag, when the reddening to NGC 300 is considered. Our faintest detected [O III] 5007 magnitude is about $27.7 \mathrm{mag}$, therefore central stars of HII regions can be detected in our images, but PN central stars are typically 2-2.5 mag fainter (Méndez et al. 1992), so are undetectable; however, it could happen that a field star is projected on a nebula, complicating the classification. Only spectroscopy can help in such a situation (see e.g., the cases discussed by Richer \& McCall 2007).
Certainly there are intruders in any sample of PN candidates. This could be the case for very compact HII regions with a faint central star or no central star at all (as could occur in compact knots embedded in HII regions). Also, very young unresolved supernova remnants (SNR) can masquerade as PNe; however, these objects are rare, so their contamination is minimal. In addition, objects from the background like unresolved Ly $\alpha$ galaxies with extremely strong Ly $\alpha$ could mimic PNe. At $z=3.126$, Ly $\alpha$ is redshifted to $5009 \AA$, which is the redshifted wavelength of [O III] 5007 for NGC 300 by considering a heliocentric radial velocity of $142 \mathrm{~km} \mathrm{~s}^{-1}$ (de Vaucouleurs et al. 1991). However, the surface density of such objects is low. In a deep search in an area of $0.27 \mathrm{deg}^{2}$, Gronwald et al. (2007) detected 162 Ly $\alpha$ emitters with monochromatic fluxes brighter than $1.5 \times 10^{-17} \mathrm{erg} \mathrm{cm}^{-2} \mathrm{~s}^{-1}$, in the $5007 \AA$ band. Of these, about 60 objects would be above our detection limit, which is $\sim 2.5 \times 10^{-17} \mathrm{erg} \mathrm{cm}^{-2} \mathrm{~s}^{-1}$. Because we sampled a field of $6.8 \times 6.8 \mathrm{arcmin}^{2}$, only three of such objects would be contaminating our sample. Thus, these contaminants would only represent a few percent of our sample, and the majority would be in the faint end of the [O III] 5007 flux distribution.

For the case of compact HII regions (cHII), ideally a second or even a third discriminating criterion would help. For instance, the excitation degree represented by the [O III] 5007 / H $\alpha$ flux ratio, which is usually larger in PNe than in HII regions, is a commonly used criterion for selecting PN candidates. Magrini et al. (2000), Ciardullo et al. (2002), and Herrmann et al. (2008) have selected those objects with [O III] $5007 / \mathrm{H} \alpha \geq 3$ as PNe. Another criterion could be to consider that cHII should be much brighter in $\mathrm{H} \alpha$ than PNe, owing to the larger amount of ionizing photons emitted by their central stars. Unfortunately, we only have [O III] 5007 images, and this latter criterion is not true for this emission line, since it depends strongly on the nebular ionization structure, and cHIIs could be bright or faint in $5007 \AA$. Then, we only depend on the absence of a visible central star and the compactness of objects to separate PN candidates from cHII, therefore we have been very careful in selecting those strictly stellar objects (size as PSF) with no detected central star as PNe. Possibly some very compact knots embedded in HII regions could be contaminating the sample. These knots have in general higher density than the surrounding nebula, thus they show low excitation and low [O III] 5007, making it difficult to detect them in an [O III] 5007 image unless they are very bright. Because of this, we consider that the contamination of our PN sample with this kind of knots represents no more than a few percentage points.

\subsection{Results from imaging}

In conclusion, from our deep search and following the criteria given above, we detected more than a hundred PN candidates, which is the largest sample obtained for NGC 300 so far. Their coordinates are presented in Cols. 2 and 3 of Table 2, and their spatial distribution is shown in Fig. 1. Interestingly, PN candidates appear concentrated mainly in the central zones of the galaxy but they are not particularly associated with the spiral structure where, on the other hand, most of the HII regions reside. There are large zones in the outer regions of the galaxy where no PN has been found. This is mainly due to the low stellar density in these zones.

We also detected a large number of extended and compact HII regions. The latter appear in our imaging as compact nebulae with a detectable central star. A brief sample of cHII were 
A\&A 547, A78 (2012)

Table 2. Photometric properties of PN candidates and compact HII regions in NGC 300.

\begin{tabular}{|c|c|c|c|c|c|c|c|c|}
\hline No. & RA (2000) & $\operatorname{Dec}(2000)$ & $m_{i}(5007)$ & Error & $\log F_{5007}{ }^{1}$ & $\log F_{5007}{ }^{2}$ & $m(5007)$ & Other ID $^{3}$, \\
\hline 1 & 005431.63 & -373904.25 & 23.45 & 0.20 & & -16.091 & $\frac{1110 \mathrm{~g}}{26.49}$ & \\
\hline 2 & 005433.17 & -373828.00 & 20.37 & 0.02 & -14.913 & -14.830 & 23.33 & $\mathrm{cHII}$ \\
\hline 3 & 005433.42 & -374431.64 & 21.03 & 0.02 & & -15.097 & 24.00 & \\
\hline 4 & 005434.85 & -374412.16 & 23.38 & 0.10 & & -16.062 & 26.42 & \\
\hline 5 & 005435.39 & -373936.00 & 18.59 & 0.01 & -14.179 & -14.099 & 21.51 & cHII, D 37 \\
\hline 6 & 005435.62 & -374116.80 & 24.04 & 0.18 & & -16.334 & 27.09 & \\
\hline 7 & 005435.75 & -374110.14 & 23.73 & 0.12 & & -16.206 & 26.77 & \\
\hline 8 & 005436.05 & -373950.80 & 19.49 & 0.01 & -14.438 & -14.468 & 22.43 & cHII, D 38 \\
\hline 9 & 005436.57 & -373858.70 & 24.70 & 0.80 & & -16.603 & 27.77 & \\
\hline 10 & 005437.55 & -373903.49 & 23.06 & 0.17 & & -15.931 & 26.09 & \\
\hline 11 & 005437.71 & -374118.98 & 24.42 & 1.41 & & -16.488 & 27.48 & \\
\hline 12 & 005437.88 & -374014.05 & 21.93 & 0.04 & -15.496 & -15.467 & 24.93 & \\
\hline 13 & 005438.13 & -373941.40 & 23.70 & 0.12 & & -16.193 & 26.74 & \\
\hline 14 & 005438.91 & -373943.20 & 20.67 & 0.01 & -14.934 & -14.951 & 23.64 & \\
\hline 15 & 005438.94 & -373843.48 & 23.74 & 0.12 & & -16.209 & 26.78 & \\
\hline 16 & 005439.59 & -374210.69 & 23.18 & 0.15 & & -15.979 & 26.21 & \\
\hline 17 & 005439.68 & -374321.40 & 22.51 & 0.08 & -15.764 & -15.703 & 25.52 & \\
\hline 18 & 005439.76 & -374134.64 & 23.37 & 0.22 & & -16.058 & 26.40 & \\
\hline 19 & 005440.04 & -374001.99 & 23.93 & 0.18 & & -16.288 & 26.98 & \\
\hline 20 & 005441.58 & -374021.40 & 22.02 & 0.10 & -15.480 & -15.505 & 25.02 & S 12 \\
\hline 21 & 005441.93 & -374043.07 & 22.69 & 0.10 & & -15.779 & 25.71 & S 15 \\
\hline 22 & 005442.23 & -374004.80 & 20.82 & 0.02 & -15.023 & -15.012 & 23.79 & S 3 \\
\hline 23 & 005443.47 & -373936.11 & 21.38 & 0.03 & & -15.242 & 24.36 & \\
\hline 24 & 005443.70 & -374151.29 & 20.93 & 0.02 & -15.069 & -15.056 & 23.90 & S 8 \\
\hline 25 & 005444.42 & -374129.40 & 20.82 & 0.02 & -14.955 & -15.012 & 23.79 & $\mathrm{~S} 2$ \\
\hline 26 & 005444.78 & -374227.72 & 22.67 & 0.08 & & -15.771 & 25.69 & \\
\hline 27 & 005445.03 & -374028.82 & 22.99 & 0.12 & & -15.902 & 26.01 & S 17 \\
\hline 28 & 005445.77 & -374130.40 & indef & & & & & S 22 \\
\hline 29 & 005445.78 & -373958.11 & 22.47 & 0.10 & & -15.687 & 25.48 & \\
\hline 30 & 005445.97 & -373752.84 & 24.06 & 0.37 & & -16.341 & 27.11 & \\
\hline 31 & 005448.05 & -373941.67 & 23.29 & 0.20 & & -16.026 & 26.33 & \\
\hline 32 & 005448.12 & -374048.57 & 24.01 & 0.37 & & -16.321 & 27.06 & S 23 \\
\hline 33 & 005448.19 & -374411.51 & 22.20 & 0.04 & & -15.578 & 25.21 & \\
\hline 34 & 005448.20 & -374341.76 & 25.74 & 0.93 & & -17.029 & 28.83 & \\
\hline 35 & 005448.38 & -373948.42 & 20.04 & 0.01 & -14.693 & -14.692 & 22.99 & \\
\hline 36 & 005449.23 & -374019.14 & 25.08 & 1.00 & & -16.757 & 28.15 & very uncertain \\
\hline 37 & 005449.44 & -374042.49 & 22.86 & 0.12 & & -15.849 & 25.88 & \\
\hline 38 & 005449.71 & -373948.43 & 22.49 & 0.10 & & -15.696 & 25.50 & \\
\hline 39 & 005451.25 & -374146.21 & 20.64 & 0.02 & -15.039 & -14.939 & 23.61 & $\mathrm{~S} 4, \mathrm{cHII}$ in $\mathrm{HII}$ \\
\hline 40 & 005452.08 & -374243.20 & 20.89 & 0.02 & -15.056 & -15.042 & 23.86 & \\
\hline 41 & 005452.14 & -374139.91 & 22.45 & 0.13 & & -15.681 & 25.46 & \\
\hline 42 & 005453.26 & -374054.41 & 22.59 & 0.10 & & -15.737 & 25.60 & S 14 \\
\hline 43 & 005453.40 & -374156.15 & 22.96 & 0.10 & & -15.889 & 25.98 & \\
\hline 44 & 005453.42 & -374028.92 & 21.90 & 0.05 & & -15.455 & 24.90 & \\
\hline 45 & 005453.82 & -373927.50 & 21.10 & 0.02 & -15.144 & -15.127 & 24.08 & \\
\hline 46 & 005454.15 & -374333.92 & 22.28 & 0.08 & & -15.610 & 25.29 & \\
\hline 47 & 005454.32 & -374232.36 & 23.61 & 0.25 & & -16.156 & 26.65 & \\
\hline 48 & 005454.91 & -374132.42 & 20.91 & 0.20 & -15.025 & -15.049 & 23.88 & S 7 \\
\hline 49 & 005454.97 & -374104.56 & 23.09 & 0.20 & & -15.943 & 26.12 & \\
\hline 50 & 005454.98 & -374311.64 & 24.45 & 0.40 & & -16.498 & 27.51 & \\
\hline 51 & 005455.33 & -374128.54 & 20.89 & 0.02 & -15.025 & -15.041 & 23.86 & S 5 \\
\hline 52 & 005455.99 & -374314.70 & 23.50 & 0.25 & & -16.111 & 26.54 & \\
\hline 53 & 005456.26 & -374029.64 & 20.17 & 0.01 & & -14.746 & 23.12 & \\
\hline 54 & 005456.83 & -373943.49 & 21.19 & 0.02 & -15.118 & -15.164 & 24.17 & \\
\hline 55 & 005456.97 & -374059.77 & 23.02 & 0.26 & & -15.914 & 26.05 & \\
\hline 56 & 005457.22 & -374122.92 & 24.75 & 0.64 & & -16.624 & 27.82 & \\
\hline 57 & 005457.42 & -374100.96 & 19.61 & 0.01 & -14.431 & -14.517 & 22.55 & cHII, D 101 \\
\hline 58 & 005458.12 & -374044.87 & 20.65 & 0.02 & -14.916 & -14.941 & 23.61 & $\mathrm{~S} 1$ \\
\hline 59 & 005458.16 & -374110.32 & 21.87 & 0.04 & & -15.443 & 24.87 & S 11 \\
\hline 60 & 005458.34 & -374116.08 & 21.39 & 0.06 & & -15.247 & 24.38 & S 13 \\
\hline 61 & 005458.48 & -374114.39 & 22.28 & 0.07 & & -15.611 & 25.29 & S 10 \\
\hline 62 & 005459.53 & -374101.46 & 22.52 & 0.08 & & -15.708 & 25.53 & S 18 \\
\hline 63 & 005459.72 & -373926.14 & 21.35 & 0.03 & -15.202 & -15.230 & 24.33 & \\
\hline 64 & 005500.47 & -373826.70 & 23.40 & 0.12 & & -16.070 & 26.44 & \\
\hline 65 & 005501.71 & -374029.39 & 21.61 & 0.04 & -15.259 & -15.336 & 24.60 & S 9 \\
\hline
\end{tabular}


Table 2. continued.

\begin{tabular}{|c|c|c|c|c|c|c|c|c|}
\hline No. & $\begin{array}{l}\text { RA (2000) } \\
\mathrm{h} \text { m s }\end{array}$ & $\begin{array}{c}\operatorname{Dec}(2000) \\
{ }^{\prime}\end{array}$ & $\begin{array}{c}m_{i}(5007) \\
\text { mag }\end{array}$ & $\begin{array}{c}\text { Error } \\
\text { mag }\end{array}$ & $\log F_{5007}{ }^{1}$ & $\log F_{5007^{2}}$ & $\begin{array}{c}m(5007) \\
\text { mag }\end{array}$ & $\begin{array}{l}\text { Other ID }{ }^{3}, \\
\text { comments }\end{array}$ \\
\hline 66 & 005502.44 & -373954.65 & 21.06 & 0.02 & -15.052 & -15.111 & 24.04 & \\
\hline 67 & 005503.12 & -374208.10 & 23.49 & 0.15 & & -16.106 & 26.52 & S 21 \\
\hline 68 & 005503.72 & -374042.49 & 23.19 & 0.17 & & -15.984 & 26.22 & S 25 \\
\hline 69 & 005503.97 & -374053.33 & 20.95 & 0.02 & -15.008 & -15.066 & 23.92 & S 6 \\
\hline 70 & 005504.04 & -374238.95 & 23.33 & 0.23 & & -16.041 & 26.36 & \\
\hline 71 & 005504.79 & -374044.70 & 23.70 & 0.15 & & -16.193 & 26.74 & \\
\hline 72 & 005504.85 & -374046.27 & 23.02 & 0.14 & & -15.916 & 26.05 & S 19 \\
\hline 73 & 005505.51 & -373829.04 & 23.53 & 0.16 & & -16.123 & 26.56 & \\
\hline 74 & 005505.77 & -374211.88 & 20.38 & 0.02 & -14.814 & -14.832 & 23.34 & \\
\hline 75 & 005505.81 & -374239.96 & 23.11 & 0.23 & & -15.949 & 26.13 & \\
\hline 76 & 005507.21 & -374142.29 & 22.82 & 0.10 & & -15.833 & 25.84 & \\
\hline 77 & 005507.49 & -374318.40 & 23.14 & 0.12 & & -15.962 & 26.16 & \\
\hline 78 & 005507.76 & -374102.01 & 23.38 & 0.13 & & -16.062 & 26.42 & \\
\hline 79 & 005507.78 & -374328.30 & indef & & & & & \\
\hline 80 & 005508.11 & -374113.22 & 23.81 & 0.17 & & -16.236 & 26.85 & \\
\hline 81 & 005508.13 & -373938.66 & 23.93 & 0.25 & & -16.288 & 26.98 & \\
\hline 82 & 005508.94 & -374140.99 & 22.76 & 0.10 & & -15.809 & 25.78 & \\
\hline 83 & 005509.33 & -374010.72 & 23.50 & 0.17 & & -16.111 & 26.54 & \\
\hline 84 & 005509.84 & -373955.42 & 24.07 & 0.34 & & -16.345 & 27.12 & \\
\hline 85 & 005510.43 & -374134.87 & 23.88 & 0.18 & & -16.266 & 26.93 & \\
\hline 86 & 005511.21 & -374220.95 & 22.96 & 0.12 & -16.088 & -15.890 & 25.99 & \\
\hline 87 & 005513.77 & -374139.23 & 18.51 & 0.02 & -14.363 & -14.065 & 21.42 & cHII, D137c \\
\hline 88 & 005513.78 & -374032.63 & 22.36 & 0.10 & -15.627 & -15.644 & 25.37 & \\
\hline 89 & 005514.14 & -374052.88 & 22.89 & 0.02 & & -15.859 & 25.91 & \\
\hline 90 & 005515.08 & -374414.5 & 19.60 & 0.02 & -14.648 & -14.513 & 22.54 & cHII \\
\hline 91 & 005515.91 & -374320.38 & 21.29 & 0.03 & -15.157 & -15.206 & 24.27 & \\
\hline 92 & 005516.65 & -374405.93 & 22.80 & 0.10 & -15.749 & -15.823 & 25.82 & \\
\hline 93 & 005521.17 & -374221.63 & 25.49 & 0.95 & & -16.926 & 28.58 & very uncertain \\
\hline 94 & 005521.18 & -374429.69 & 23.68 & 0.17 & -16.252 & -16.186 & 26.73 & \\
\hline 95 & 005521.62 & -374022.44 & 23.55 & 0.11 & -16.271 & -16.133 & 26.59 & \\
\hline 96 & 005522.54 & -374216.51 & 21.60 & 0.03 & -15.263 & -15.333 & 24.59 & \\
\hline 97 & 005523.68 & -374455.43 & 21.63 & 0.03 & -15.273 & -15.342 & 24.62 & \\
\hline 98 & 005524.06 & -374604.26 & 24.50 & 0.30 & & -16.522 & 27.56 & \\
\hline 99 & 005524.49 & -374257.43 & 24.69 & 0.35 & & -16.597 & 27.75 & \\
\hline 100 & 005525.87 & -374353.87 & 23.62 & 0.17 & & -16.160 & 26.66 & \\
\hline 101 & 005526.39 & -374045.05 & 24.17 & 0.18 & -16.485 & -16.387 & 27.23 & \\
\hline 102 & 005527.20 & -374343.55 & 19.91 & 0.02 & -14.672 & -14.640 & 22.86 & cHII \\
\hline 103 & 005527.49 & -374055.13 & 19.99 & 0.02 & -14.603 & -14.670 & 22.94 & \\
\hline 104 & 005528.88 & -374440.13 & 21.66 & 0.03 & -15.273 & -15.355 & 24.65 & \\
\hline 105 & 005529.15 & -374453.48 & 23.06 & 0.16 & & -15.932 & 26.09 & \\
\hline 106 & 005529.93 & -374444.23 & 23.90 & 0.18 & & -16.273 & 26.94 & \\
\hline 107 & 005529.99 & -374142.18 & 24.02 & 0.20 & & -16.324 & 27.07 & \\
\hline 108 & 005530.30 & -374115.68 & 20.20 & 0.03 & -14.679 & -14.759 & 23.16 & \\
\hline 109 & 005530.53 & -374359.16 & 21.16 & 0.03 & -15.023 & -15.153 & 24.14 & \\
\hline 110 & 005530.90 & -374357.04 & 22.96 & 0.10 & & -15.891 & 25.99 & \\
\hline 111 & 005535.42 & -374421.93 & 24.14 & 0.25 & & -16.373 & 27.19 & \\
\hline 112 & 005539.13 & -373935.24 & 24.28 & 0.25 & & -16.431 & 27.34 & \\
\hline
\end{tabular}

Notes. ${ }^{(1)}$ Flux from spectroscopy, in erg $\mathrm{cm}^{-2} \mathrm{~s}^{-1}$. (2) Flux calculated from the equation in Fig. 2, in $\mathrm{erg} \mathrm{cm}^{-2} \mathrm{~s}^{-1}$. (3) S\#: IDs for PNe from Soffner et al. (1996). D\#: IDńs for compact HII regions (cHII) from Deharveng et al. (1998).

chosen to be analyzed spectroscopically, together with a sample of PN candidates (see Sect. 2.2). Some of our cHII have been previously reported in the catalog by Deharveng et al. (1988). The physical conditions and abundances of these regions will be discussed in a subsequent paper in comparison with the spectroscopic properties of the PN candidates.

We rediscovered 25 of the $34 \mathrm{PN}$ candidates reported by Soffner et al. (1996), who analyzed some central zones of NGC 300, all of which lie inside our central zone. The 25 rediscovered objects correspond to the brightest PN candidates of Soffner et al., numbered in their work from 1 to 23 and their objects \#25 and \#27. In this work, we confirmed the PN nature of all of them except their object \#4, which from our spectroscopy turned out to be a compact HII region (our object \#39 in Table 2), their objects \#16 and \#20, which appear as faint and diffuse nebulae (therefore they are not PNe) and their object \#27, which is a faint star. The last three are not included in our list. The rest of the Soffner et al. objects definitely do not appear in our images, even when we searched carefully in the positions of the alleged $\mathrm{PNe}$. Our [O III] recombined images are deeper than the ones by Soffner et al. because ours have $12.5 \mathrm{~min}$ of exposure time with the VLT, which is equivalent to more than one hour with a $3.6-\mathrm{m}$ telescope, which were the exposure time and telescope used by Soffner et al. Besides this, our images were obtained under much 
better seeing conditions (better than $0.9^{\prime \prime}$ against the $1^{\prime \prime}$ up $2^{\prime \prime}$ reported by Soffner et al.), and they have better spatial resolution, so we were able to detect much fainter objects than object \#25 of Soffner et al. We therefore conclude that their objects \#s 24, 26, and from \#28 to \#34 are possible misidentifications. It is worth noticing that the missing objects would belong to their field $\mathrm{W}$, which is very crowded, and it was observed with only half an hour of exposure time, therefore misidentifications are not rare. Soffner et al. (1996) ID's are included in Col. 9 of our Table 2.

For all the PN candidates (104 objects) and eight compact HII regions, we performed a comparative photometry measuring the instrumental magnitudes in the [O III] 5007 on-band images. The IRAF task digiphot.apphot.phot was used. The typical PSF of the images is 2.9 pix (FWHM), thus an aperture of 5 pix radius (equivalent to $0.63^{\prime \prime}$ ) was used to integrate the object flux, and the sky was subtracted from a ring of 5 pix radius and width of 2 pix around the object. The instrumental magnitudes $m_{i}(5007)$ and their errors are presented in Cols. 4 and 5 of Table 2.

\section{3. [O III] calibration}

The most confident way to distinguish among different types of emission nebulae is by their spectral analysis. However, this is expensive in terms of telescope time because these objects are very faint in external galaxies. Our follow-up spectroscopy included more than 40 nebulae. From these data we confirm the PN nature of 19 PN candidates in the central zone and 13 in the outskirts. The confirmed PNe are those objects having a measured [O III] 5007 flux in Col. 6 of Table 2. Several compact HII regions were also observed. Their calibrated [O III] $5007 \AA$ Al fluxes are also listed in Col. 6 of Table 2.

Figure 2 presents the relation between our instrumental magnitudes $m_{i}(5007)$ and the logarithm of spectroscopic fluxes for all the analyzed PN candidates and compact HII regions. It is notable that a very good linear correlation can be fitted to the data, through an interval of five magnitudes. The two brightest objects lying slightly below the correlation are the cHIIs \#5 and \#87 of Table 2. These cHIIs are slightly extended and some flux might have been lost in the spectroscopic slit of $1^{\prime \prime}$ width. At the other extreme, the four faintest objects that do not fit very well are very faint $\mathrm{PNe}$ for which the instrumental magnitudes have relatively large errors $(\Delta m \geq 0.2 \mathrm{mag})$. To discard these objects produces no significant difference in the correlation.

The linear fit shown in Fig. 2 was used to calculate calibrated [O III] 5007 fluxes for all the objects from their instrumental magnitudes. The fluxes are presented in Col. 7 of Table 2, and in Col. 8 we list the [O III] 5007 apparent magnitudes, calculated as $m(5007)=-2.5 \log F_{5007}-13.74$, with $F_{5007}$ in $\mathrm{erg} \mathrm{cm}^{-2} \mathrm{~s}^{-1}$ (Allen 1973).

\section{The PN sample and the planetary nebulae luminosity function}

There are 66 PN candidates in the central zone and 34 in the outskirts, for which confident [O III] 5007 apparent magnitudes were determined. Although the number of objects is small for a reliable statistics in order to derive the peak magnitude of the PNLF and the distance to NGC 300, a rough [O III] 5007 luminosity function (PNLF) can be constructed for the whole sample and for both zones. The PNLF helps to obtain a better understanding of the PN populations in a galaxy; thus, with this purpose, in the following sections we construct and analyze the differential PNLF.

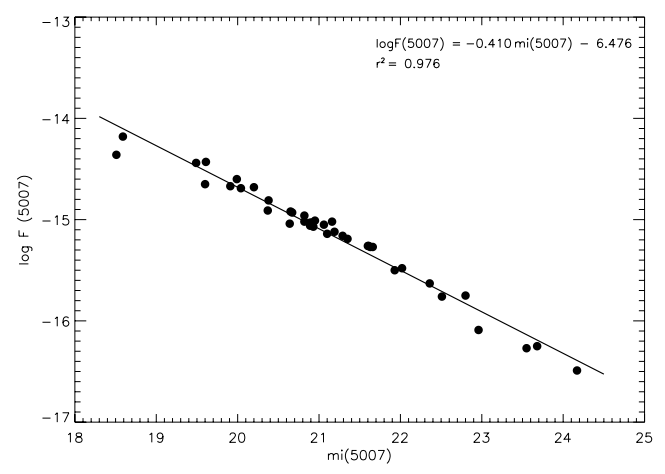

Fig. 2. Relation between spectroscopic [O III] 5007 fluxes, $F(5007)$, and instrumental magnitudes $m_{\mathrm{i}}(5007)$ for all the spectroscopically analyzed objects (PN candidates and compact HII regions are included). The parameters of the linear fit are shown in the figure. The objects lying slightly out of the correlation are discussed in the text.

\subsection{The PNLF behavior}

In our experience, the shape of the PNLF is very sensitive to the selection of the bin size, in particular when the sample is small. With a bin size too small, some bins could have very few objects and a very sparse PNLF could be obtained. The effect of the bin size can be observed, for instance, in the two PNLFs built for the SMC PNe by Jacoby et al. (1990) and Jacoby \& De Marco (2002); the first one, with a bin size of $0.2 \mathrm{mag}$, is very sparse and the other, with a bin of $1 \mathrm{mag}$, shows a shape similar to the empirical PNLF. A full discussion of the effect of the bin size on the PNLF will be published elsewhere (Rodríguez-González et al., in prep.).

Thus, due to the low number of objects in our samples we were cautious with the bin size selection, and carried out several proofs for the bin size (defined as $b s=\left(m_{\max }-m_{\min }\right) / N b i n s$, where $b s$ is the bin size, $m_{\max }$ and $m_{\min }$ are the maximum and minimum magnitude values in the sample, and Nbins is the number of bins that we assign to our distribution), in order to obtain an observed PNLF with a shape similar to the empirical one (Eq. (2)). Finally, from our [O III] 5007 calibrated magnitudes for the PN sample we computed the observed PNLFs for the whole sample and for each region (inner and outskirts) using bin sizes of $1.16 \mathrm{mag}, 1.12 \mathrm{mag}$, and $1.12 \mathrm{mag}$ respectively. Therefore, for all the cases, the sample is divided into five bins. The three observational PNLFs are presented in Fig. 3. The error bar assigned to each bin represents the statistical Poissonian error.

The fit of the empirical PNLF to the observed PNLF (computed by using a fitting scheme based on the LevenbergMarquardt technique that uses a $\chi^{2}$ minimization) was calculated by converting the usual differential luminosity function for absolute magnitudes given in Eq. (1) (Jacoby 1989; Ciardullo et al. 1989),

$N(M) \propto \mathrm{e}^{0.307 M_{5007}}\left(1-\mathrm{e}^{3\left(M_{5007}^{*}-M_{5007}\right)}\right)$

to a luminosity function in apparent magnitudes (Eq. (2))

$N(m)=N \mathrm{e}^{-0.307 \mu} \mathrm{e}^{0.307 m_{5007}}\left(1-\mathrm{e}^{3\left(m_{5007}^{*}-m_{5007}\right)}\right)$,

where $N$ is a normalization constant, $\mu$ the apparent distance modulus, $\mu=5 \log d-5+A_{5007}$, and $M_{5007}^{*}$ and $m_{5007}^{*}$ are the absolute and apparent peak magnitudes of the luminosity function.

The observed PNLF for the whole sample (Fig. 3 left) seems complete in the first four bins (the fifth one already shows incompleteness) so we used them for fitting the empirical PNLF 

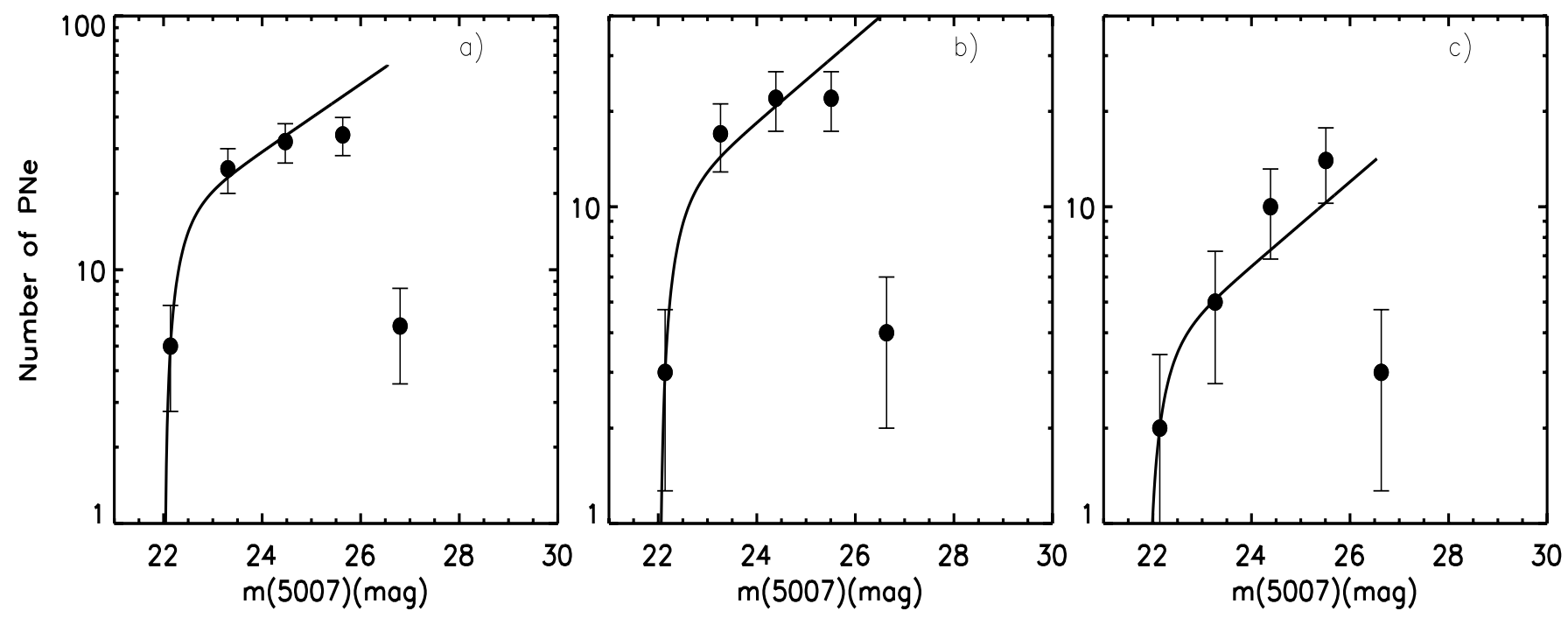

Fig. 3. Observed PN luminosity function for: a) the whole sample, b) the PNe in the central zone, and c) the PNe in the outskirts of NGC 300. Bin sizes are described in the text. The errors bars correspond to the Poissonian error. Solid lines represent our best fit of the empirical PNLF for the four brightest bins in each case.

(Eq. (2)). A Kolmogorov-Smirnov test applied to this fit shows that both functions (observed and empirical) are equal within the first four bins, with a reliance level of $99.96 \%$. The PNLF adjusted for the whole sample returns a value $m^{*}(5007)=22.019 \pm$ $0.022 \mathrm{mag}$, and a value $N \mathrm{e}^{-0.307 \mu}=0.0187 \pm 0.0021$.

For the central zone, the observed PNLF (Fig. 3 middle) also seems complete in the first four bins and this PNLF is very similar to the one for the whole sample because it contains the majority of the total PN candidates. Again a Kolmogorov-Smirnov test applied to this fit indicates that the observed and empirical functions are equal within the first four bins, with a reliance level of $99.96 \%$. The PNLF adjusted for the central sample returns values of $m^{*}(5007)=22.02 \pm 0.03 \mathrm{mag}$, and $N \mathrm{e}^{-0.307 \mu}=$ $0.0118 \pm 0.00158$, completely similar to values of the whole sample.

The sample of the outskirts (Fig. 3 right) has only 34 objects and the empirical PNLF was adjusted to the first four bins which seem complete. Also a Kolmogorov-Smirnov test for this fit predicts that both curves are the same, with a reliance of $99.96 \%$. The $\mathrm{m}^{*}(5007)$ value returned is $21.88 \pm 0.07 \mathrm{mag}$, and $N \mathrm{e}^{-0.307 \mu}=0.0042 \pm 0.00105$, which is slightly lower than the previous values, but it could be consider similar within uncertainties. Therefore we find no evidence of a significant change in the PNLF with the galactic position in NGC 300.

The behavior of the PNLF is normal, in the sense that none presents any noticeable dip such as the ones found in the irregular galaxies, e.g., the SMC (Jacoby \& De Marco 2002), the LMC (Reid \& Parker 2010), and NGC 6822 (Hernández-Martínez \& Peña 2009), which have been interpreted as due to the existence of a younger population of PNe in which the central star evolution proceeds very quickly.

Since the top three magnitudes of the PNLF of the whole sample are significantly complete and this is the distancesensitive segment, it is tempting to use our fit result for a tentative estimate of the distance to NGC 300. As already said, the apparent peak magnitude returned by our best fit is $m^{*}(5007)=$ $22.019 \pm 0.022$. This value should be dereddened for the foreground extinction. It is known that the external reddening towards NGC 300 is very low. The estimated values fluctuate between $E(B-V)=0.013$ (Schlegel et al. 1998) and
$E(B-V)=0.096$ (Gieren et al. 2005). These values translate into an absorption $A_{5007}$ of 0.05 and $0.3 \mathrm{mag}$, respectively. By adopting $A_{5007} \sim 0.20 \mathrm{mag}$ and assuming a peak absolute magnitude $M^{\star}(5007)=-4.47$ as derived from Fig. 5 of Ciardullo et al. (2002) for the NGC 300 central metallicity (adopted to be $12+\log \mathrm{O} / \mathrm{H}=8.57$, Bresolin et al. 2009), we obtain a distance modulus $m-M=26.29_{-0.22}^{+0.12}$. The error bar includes the formal error of the fit $\left(0.022 \mathrm{mag}\right.$ ), the error in $A_{5007}$ (about $0.1 \mathrm{mag}$ ), and on the bright side, we have added a $10 \%$ error due to possible flux losses in the spectroscopic slit. Our derived distance is in good agreement with the distance modulus of $26.37 \pm 0.08$ reported from Cepheids by Gieren et al. (2005). This confirms the robustness of the method of deriving distances to external galaxies through the bright cut-off of the PNLF.

\section{Conclusions}

From deep [O III] 5007 on-band and off-band imaging, performed with VLT FORS 2 to search for line emission objects in two zones of the spiral galaxy NGC 300, we detected more than a hundred PN candidates, and many compact and extended HII regions. From our follow-up spectroscopy, the 32 candidates analyzed out of the 104 objects in the sample have been confirmed as true planetary nebulae, therefore we are confident that our methods for selecting $\mathrm{PNe}$ are appropriate.

The instrumental [O III] 5007 mag derived from the imaging were calibrated by using the results from our spectroscopy. This allowed us to determine calibrated apparent [O III] 5007 magnitudes for our PN candidates and some compact HII regions.

We built the observed [O III] 5007 PNLF for the whole PN sample, as well as for the samples of the central zone and the outskirts. All the PNLFs are similar within uncertainties and they appear normal, in that they show no dip as the dips reported for the PNLFs of some irregular galaxies (the LMC, the SMC, and NGC 6822). We fit the empirical PNLF to our data, obtaining a very good fit for the three brightest magnitudes in all the cases; in particular, the whole sample and the central one return the same results.

Our best fit for the three brightest magnitudes of the observed PNLF for the whole sample allows us to determine a 
distance modulus $m-M=26.29_{-0.22}^{+0.12} \mathrm{mag}$, which is in good agreement with the value of $26.37 \pm 0.08$ mag derived by Gieren et al. (2005) for Cepheid stars in NGC 300.

Acknowledgements. Invaluable comments by G. Stasińska are deeply appreciated. M. Peña is grateful to DAS, Universidad de Chile, for hospitality during a sabbatical stay when part of this work was done. L.H.-M. benefited from the hospitality of the Departamento de Astronomía, Universidad de Chile. We are grateful to Dr. R. Napiwotzki and to an anonymous referee for providing comments that helped us to improve the manuscript. L.H.-M., J.R.-P., and M.P.-G. received scholarships from CONACYT-México. M.P. gratefully acknowledges financial support from FONDAP-Chile and DGAPA-UNAM. This work received financial support from grants \#43121 (CONACYT-México), and IN-105511(PAPIIT DGAPA-UNAM).

\section{References}

Allen, C. W. 1973, Astrophysical Quantities (London Athlone) Appenzeller, I., Fricke, K., Fürtig, W., et al. 1998, The Messenger, 94, 1 Bresolin, F., Gieren, W., Kudritzki, R.-P., et al. 2009, ApJ, 700, 309

Ciardullo, R., Ford, H. C., Neil, J. D., Jacoby, G. H., \& Shafter, A. W. 1987, ApJ, 318,520

Ciardullo, R., Jacoby, G. H., Ford, H. C., \& Neill, J. D. 1989, ApJ, 339, 53

Ciardullo, R., Feldmeier, J. J., Jacoby, G. H., et al. 2002, ApJ, 577, 31 de Vaucouleurs, G., de Vaucouleurs, A., Corwin Jr., H. G., et al. 1991, Third Reference Catalogue of Bright Galaxies, version 3.9 (New York: Springer-Verlag)

Deharveng, L., Caplan, J., Lequeux, J., et al. 1988, A\&ASS, 73, 407

Gieren, W., Pietrzyński, G., Soszynski, I., et al. 2005, ApJ, 628, 695

Gronwald, C., Ciardullo, R., Hickey, T., et al. 2007, ApJ, 667, 79

Hernández-Martínez, L., \& Peña, M. 2009, A\&A, 495, 447

Herrmann, K. A., Ciardullo, R., Feldmeier, J. J., \& Vinciguerra, M. 2008, ApJ, 683,630

Jacoby, G. H. 1989, ApJ, 339, 39

Jacoby, G. H., \& De Marco, O. 2002, AJ, 123, 269

Jacoby, G. H., Walker, A. R., \& Ciardullo, R. 1990, 365, 471

Magrini, L., Corradi, R. L. M., Mampaso, A., \& Perinotto, M. 2000, A\&A, 355, 713

Martins, F., Schaerer, D., \& Hillier, D. J. 2005, A\&A, 436, 1049

Méndez, R. H., Kudritzki, R., \& Herrero, A. 1992, A\&A, 260, 329

Peña, M., Richer, M. G., \& Stasinska, G. 2007, A\&A, 466, 75

Reid, W. A., \& Parker, Q. A. 2010, MNRAS, 405, 1349.

Richer, M. G., \& McCall, M. 2007, ApJ, 658, 328

Rizzi, L., Méndez, R. H., \& Gieren, W. 2006, Planetary Nebulae in our Galaxy and Beyond, eds. M. J. Barlow, \& R. H. Méndez (Cambridge University Press), Proc. IAU Symp., 234, 493

Schlegel, D. J., Finkbeiner, D. P., \& Davis, M. 1998, ApJ, 500, 525

Soffner, T., Méndez, R. H., Jacoby, G. H., et al. 1996, A\&A, 306, 9

Stasińska, G., Peña, M., Bresolin, F., \& Tsamis, Y. G. 2012, A\&A, submitted

Urbaneja, M. A., Herrero, A., Bresolin, F., et al. 2005, ApJ, 622, 862 